\title{
Randomness Effect on Multicritical Phenomena in Double-Exchange Systems
}

\author{
Yukitoshi Motome ${ }^{\mathrm{a}}$, Nobuo Furukawa ${ }^{\mathrm{b}}$, Naoto Nagaosa ${ }^{\mathrm{a}, \mathrm{c}, \mathrm{d}}$ \\ a Tokura Spin SuperStructure Project (SSS), ERATO, Japan Science and Technology Corporation (JST), c/o National \\ Institute of Advanced Industrial Science and Technology (AIST), Tsukuba, Ibaraki 305-8562, Japan \\ b Department of Physics, Aoyama Gakuin University, Setagaya-ku, Tokyo 157-8572, Japan \\ c Department of Applied Physics, University of Tokyo, Bunkyo-ku, Tokyo 113-8656, Japan \\ d Correlated Electron Research Center (CERC), AIST, Tsukuba, Ibaraki 305-8562, Japan
}

\begin{abstract}
Double-exchange model interacting with adiabatic phonons is studied in the presence of randomness by using the Monte-Carlo method and the systematic size-scaling analysis. A bicritical behavior is found between the ferromagnetic metal and the charge-ordered insulator. We find the distinct response to the randomness between these two states, which agrees well with the experimental results in the colossal magnetoresistance manganites.
\end{abstract}

Key words: double-exchange model, bicritical phenomena, randomness, colossal magnetoresistance manganites PACS:

Phase competition and randomness play a key role in the colossal magnetoresistance (CMR) phenomena in manganese oxides $[1,2]$. The former phase competition between the ferromagnetic metal (FM) and the charge-ordered insulator (CO) leads to large fluctuations near the multicritical point [3]. The latter randomness suppresses the transition temperatures above which CMR is much enhanced [4]. Theoretically, except for a phenomenological argument [5], this multicritical phenomenon has not been fully investigated thus far. Therefore, it is strongly desired to study a realistic model for this phenomenon including the metal-insulator transition.

In this work, we study the multicritical phenomena in the double-exchange (DE) model interacting with phonons whose Hamiltonian reads

$$
\begin{aligned}
H= & -t \sum_{\langle i j\rangle}\left(1+S_{i} S_{j}\right)\left(c_{i}^{\dagger} c_{j}+\text { h.c. }\right) \\
& -g \sum_{i} c_{i}^{\dagger} c_{i} x_{i}+\frac{1}{2} \sum_{i} x_{i}^{2}+\frac{\lambda}{2} \sum_{\langle i j\rangle} x_{i} x_{j} \\
& +\sum_{i} \varepsilon_{i} c_{i}^{\dagger} c_{i}+\gamma M^{2} X^{2} .
\end{aligned}
$$

Here, the first line is the DE part which is derived in the limit of the large Hund coupling with the Ising symmetry $\left(S_{i}= \pm 1\right)[6]$, and the second line represents the electron-phonon coupling and the elastic energy of phonons. We consider the classical phonon in the breathing mode. The $\lambda$ term is for the cooperative distortion of the $\mathrm{MnO}_{6}$ tetrahedra. The first term in the last line denotes the random on-site potential. We consider here the 

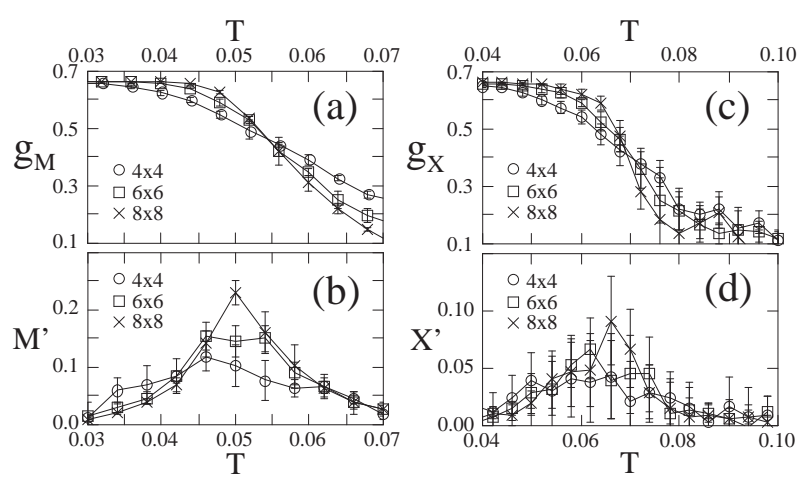

Fig. 1. The Binder parameter and the numerical derivatives of the temperature dependence of the order parameter. (a) and (b) are for FM at $g=0.8$, and (c) and (d) are for CO at $g=1.2$, respectively. Critical temperatures are indicated by the crossing of the Binder parameter in (a) and (c), and the peak of the numerical derivatives in (b) and (d).

binary-type distribution $\varepsilon_{i}= \pm \Delta$. The last term is a Gintzburg-Landau-type term which describes the competition between FM of the DE origin and CO of the checker-board type, whose order parameters are denoted as $M$ and $X$, respectively. We consider that this term mimics the elements beyond this simple model such as the orbital degeneracy and the antiferromagnetic coupling between localized spins. We consider model (1) at the electron density $n=0.5$ on the square lattice. We set the halfbandwidth $W=4 t=1$ as an energy unit, and take $\lambda=0.1$ and $\gamma=1$ in the following.

We study the thermodynamics in model (1) by MC method [7]. We have typically 10000 samplings for measurement after 1000 steps for thermalization in the absence of the randomness. In the presence of the randomness, the measurement is typically performed 1000 times for a configuration of the random potential $\left\{\varepsilon_{i}\right\}$, and the results are random-averaged for typically 16 different configurations. The transition temperatures for FM and $\mathrm{CO}$ are determined by the Binder parameter as well as the inflection point of the temperature dependence of each order parameter. Figure 1 shows a part of these analyses $(\Delta=0)$. Both results consistently indicate the emergence of the long-range ordering.

Figure 2 displays the phase diagram of model (1). In the pure case $\Delta=0$, the phase diagram shows the bicritical behavior where FM and CO

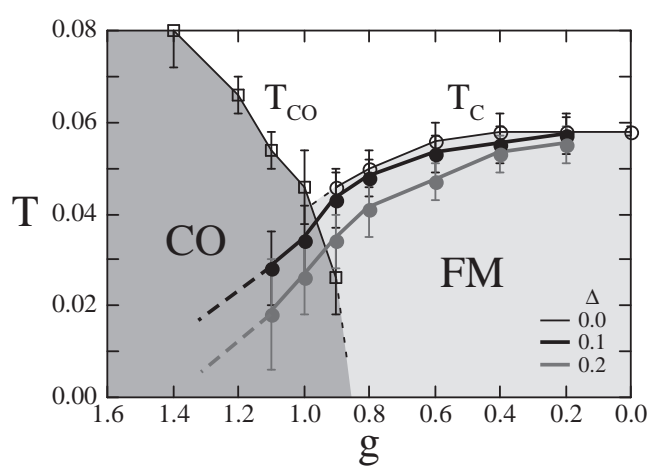

Fig. 2. Phase diagram of model (1).

phases touch with each other at $g \simeq 0.9$. When we introduce the randomness, these two phases show a distinct response; FM is rather robust whereas $\mathrm{CO}$ is surprisingly fragile to the randomness. Consequently, the CO phase is destroyed rapidly, and instead the FM state extends to larger- $g$ regions. This is a consequence of the phase competition near the bicritical point; if one phase diminishes, the other takes over. These aspects well agrees with the experimental results in the CMR manganites [4]. Therefore, we believe that model (1) captures the essential physics of this multicritical phenomenon in manganites. In experiments, the enhanced CMR is observed near the region where FM replaces CO [4]. Transport property and electronic structure in the present model will provide a key to understand the mechanism of CMR.

References

[1] Y. Tokura and N. Nagaosa, Science 288, 462 (2000).

[2] E. Dagotto, T. Hotta, and A. Moreo, Phys. Rep. 344, 1 (2001) and references therein.

[3] Y. Tomioka and Y. Tokura, Phy. Rev. B 66, 104416 (2002).

[4] D. Akahosi et al., to be published in Phys. Rev. Lett.; T. Nakajima et al., J. Phys. Soc. Jpn. 71, 2843 (2002); Y. Tomioka et al., preprint.

[5] A. Moreo, S. Yunoki, and E. Dagotto, Science 283, 2034 (1999); J. Burgy et al., Phys. Rev. Lett. 87, $277202(2001)$.

[6] Y. Motome and N. Furukawa, J. Phys. Soc. Jpn. 70, 1487 (2001).

[7] S. Yunoki et al., Phys. Rev. Lett. 80, 845 (1998). 